\title{
Multiplexed Opsonophagocytic Killing Assay
}

National Cancer Institute

\section{Source}

National Cancer Institute. Multiplexed Opsonophagocytic Killing Assay. NCI Thesaurus.

Code 147468.

An assay technique that measures the opsonophagocytic killing activity of antibodies against multiple serotypes in a single serum assay. 